\title{
Shear-Induced Transitions between a Planar Lamellar Phase and Multilamellar Vesicles: Continuous versus Discontinuous Transformation
}

\author{
B. Medronho, ${ }^{*}{ }^{\dagger, *}$ S. Shafaei,${ }^{\S}$ R. Szopko, ${ }^{\S}$ M. G. Miguel, ${ }^{\dagger}$ U. Olsson, ${ }^{\star}$ and C. Schmidt ${ }^{\S}$ \\ Department of Chemistry, University of Coimbra, 3004-535 Coimbra, Portugal, Physical Chemistry 1, \\ Center of Chemistry and Chemical Engineering, Lund University, Box 124, 22100 Lund, Sweden, and \\ Department of Chemistry, University of Paderborn, Warburger Str. 100, D-33098 Paderborn, Germany
}

Received January 31, 2008. Revised Manuscript Received April 14, 2008

\begin{abstract}
The shear-induced transitions between an oriented lamellar phase and shear-induced multilamellar vesicles (MLVs) in a nonionic surfactant system were studied by deuterium rheo-NMR spectroscopy as a function of time in start-up experiments at several temperatures and shear rates. By starting from an initial state of oriented lamellae and observing the transformation to the final steady state of MLVs and vice-versa, two different mechanisms were found, depending on the direction of the transition and the initial state. The transition is continuous when MLVs are formed, starting from the oriented lamellar phase. On the other hand, a discontinuous nucleation-and-growth process with a coexistence region is observed when transforming MLVs into an oriented lamellar phase.
\end{abstract}

\section{Introduction}

Surfactants in solution display a rich phase behavior. Phase diagrams of nonionic surfactants, for instance, of the $\mathrm{C}_{n} \mathrm{E}_{m}$ type, where $n$ is the number of carbon atoms in the hydrocarbon chain and $m$ is the number of ethylene oxide units in the headgroup, present a great variety of structures, depending on temperature and concentration. ${ }^{1-3}$ For concentrated surfactant solutions in which bilayer phases are preferentially formed, two topologically different organizations of the bilayers are possible: the lamellar, or $\mathrm{L}_{\alpha}$, phase, which consists of stacks of bilayers with longrange order and is optically birefringent, and the "sponge", or $\mathrm{L}_{3}$, phase that is a network of randomly multiconnected membranes. ${ }^{4,5}$ Often the structure of the lamellar phase does not consist of stacks of planar layers but of spherulites or multilamellar vesicles (MLVs), which are often referred to as onions and may be observed by optical microscopy. 6

MLVs often form when a lamellar phase is subject to shear flow, ${ }^{8}$ and this shear-induced transition has received much attention. $^{9-12}$ Shear-induced MLVs may be stable for a long time, but they do not correspond to the thermodynamic-

* To whom correspondence should be addressed. E-mail: Bruno.Medronho@ fkem1.lu.se.

University of Coimbra.

* Lund University.

$\S$ University of Paderborn.

(1) Chernik, G. G. Curr. Opin. Colloid Interface Sci. 2000, 4, 381.

(2) Jönsson, B.; Lindman, B.; Holmberg, K.; Kronberg, B. Surfactants and Polymers in Aqueous Solution, 2nd ed.; John Wiley \& Sons: New York, 2003.

(3) Mitchell, D. J.; Tiddy, G. J. T.; Waring, L.; Bostock, T.; McDonald, M. P. J. Chem. Soc., Faraday Trans. 1983, 79, 975. 1335.

4) Porte, G.; Appell, J.; Bassereau, P.; Marignan, J. J. Phys. (Paris) 1989, 50,

(5) Anderson, D.; Wennerstrom, H.; Olsson, U. J. Phys. Chem. 1989, 93, 4243 .

(6) Mandell, L.; Ekwall, P. Acta Polytech. Scand., Chem. Incl. Metall. Ser. 1968, 74 , I

(7) Fontell, K.; Mandell, L.; Lehtinen, H.; Ekwall, P. Acta Polytech. Scand., Chem. Incl. Metall. Ser. 1968, 74, III

(8) Diat, O.; Roux, D.; Nallet, F. J. Phys. II 1993, 3, 1427.

(9) Richtering, W. Curr. Opin. Colloid Interface Sci. 2001, 6, 446.

(10) Mortensen, K. Curr. Opin. Colloid Interface Sci. 2001, 6, 140.

(11) Butler, P. Curr. Opin. Colloid Interface Sci. 1999, 4, 214.

(12) Berni, M. G.; Lawrence, C. J.; Machin, D. Curr. Opin. Colloid Interface Sci. 2002, 98, 217. equilibrium structure. ${ }^{13}$ This was demonstrated by Bergmeier et al. for an example of a lamellar phase, which can be prepared without shearing the sample in a mixing process. ${ }^{14}$ If mixing the components of this system, in a conventional way, vesicles seem to form spontaneously, whereas the $\mathrm{L}_{\alpha}$ phase consists of planar layers when the phase is formed from the $\mathrm{L}_{3}$ phase via a chemical reaction. Diat and co-workers ${ }^{8}$ have found that, by shearing a lamellar phase at a constant shear rate, $\dot{\gamma}$, a defect structure of densely packed MLVs, which are deformed to a polyhedral shape, ${ }^{15}$ is generated. These vesicles may have a narrow size distribution, and in some cases they pack into an ordered structure. ${ }^{16-18}$ Their size is controlled by the shear rate: the vesicle diameter scales approximately with $\dot{\gamma}^{-1 / 2}$. 8,19 Although the creation of MLVs by shearing a lyotropic lamellar phase has already been documented by several authors for a large variety of surfactants, ${ }^{8,16-41}$ neither the reason nor the mechanism of their formation is understood in detail.

(13) Gradzielski, M. J. Phys.: Condens. Matter 2003, 15, 655.

(14) Bergmeier, M.; Hoffmann, H.; Thunig, C. J. Phys. Chem. B 1997, 101, 5767.

(15) Gulik-Krzywicki, T.; Dedieu, J. C.; Roux, D.; Degert, C.; Laversanne, R. Langmuir 1996, 12, 4668.

(16) Diat, O.; Roux, D.; Nallet, F. Phys. Rev. E 1995, 51, 3296.

(17) Sierro, P.; Roux, D. Phys. Rev. Lett. 1997, 78, 1496.

(18) Le, T. D.; Olsson, U.; Mortensen, K. Phys. Chem. Chem. Phys. 2001, 3, 1310 .

(19) Müller, S.; Börschig, C.; Gronski, W.; Schmidt, C.; Roux, D. Langmuir 1999, 15,7558 .

(20) Roux, D.; Nallet, F.; Diat, O. Europhys. Lett. 1993, 24, 53.

(21) Soubiran, L.; Coulon, C.; Sierro, P.; Roux, D. Europhys. Lett. 1995, 31, 243.

(22) Bergenholtz, J.; Wagner, N. J. Langmuir 1996, 12, 3122.

(23) Läuger, J.; Weigel, R.; Berger, K.; Hiltrop, K.; Richtering, W. J. Colloid Interface Sci. 1996, 181, 521.

(24) Lukaschek, M.; Müller, S.; Hansenhindl, A.; Grabowski, D. A.; Schmidt, C. Colloid Polym. Sci. 1996, 274, 1.

(25) Van der Linden, E.; Hogervorst, W. T.; Lekkerkerker, H. N. W. Langmuir 1996, $12,3127$.

(26) Panizza, P.; Colin, A.; Coulon, C.; Roux, D. Eur. Phys. J. B 1998, 4, 65.

(27) Schmidt, G.; Müller, S.; Schmidt, C.; Richtering, W. Rheol. Acta 1999, $38,486$.

(28) Zipfel, J.; Berghausen, J.; Lindner, P.; Richtering, W. J. Phys. Chem. B 1999, 103, 2841 .

(29) Zipfel, J.; Nettesheim, F.; Lindner, P.; Le, T. D.; Olsson, U.; Richtering, W. Europhys. Lett. 2001, 53, 335.

(30) Burgemeister, D.; Schmidt, C. Prog. Colloid Polym. Sci. 2002, 121, 95.

(31) Courbin, L.; Pons, R.; Rouch, J.; Panizza, P. Europhys. Lett. 2003, 61, 275. 
The dynamic out-of-equilibrium transitions between different shear-induced states may be continuous or discontinuous. ${ }^{42}$ The transition from aligned layers at low shear rates to onions at intermediate shear rates was reported as being continuous. ${ }^{8,20,21,29,38}$ The continuous nature of the formation of close packed MLVs from flow-aligned layers observed upon increasing shear rate at constant temperature was confirmed for several surfactant systems by deuterium NMR spectroscopy. ${ }^{19,24,35}$ On the other hand, a second transition observed in some surfactant systems at high shear rates, where MLVs transform into another state of aligned layers, was found to be discontinuous. ${ }^{8,20}$ Recently, Wilkins and Olmsted have shown that the lamellarto-onion transition for the quaternary system of sodium dodecyl sulfate (SDS), water, pentanol, and dodecane exhibits vorticity shear banding. ${ }^{36}$ They found this transition to be discontinuous between the two shear thinning regimes, corresponding to the low-stress state of oriented lamellae and the more viscous shearinduced MLV state. In fact, the material was macroscopically separated into bands of lamellae and onions stacked in the vorticity direction.

There has been considerable research and work concerning the transition from the normal $L_{\alpha}$ phase to MLVs. However, the reverse transition (MLVs to $L_{\alpha}$ ) has not yet received the same attention. It is important to note that the lamellar-onion transition is reversible in the following sense. When applying shear to a lamellar phase, there is a transiton to onions if the shear rate is sufficiently high. From this onion state, one may then return to a planar lamellar state by shearing with a sufficiently low shear rate. In this paper, we will demonstrate that there is a fundamental difference, depending on the direction of the transition. We will argue about the possibility of two different mechanisms (continuous and discontinuous) when start-up experiments are performed with different starting conditions (MLVs or planar lamellae). For these investigations, the nonionic surfactant system $\mathrm{C}_{10} \mathrm{E}_{3}$ /water was chosen, since its shear-induced onion formation has been well-documented. ${ }^{28,37-41}$ The shear diagram of an aqueous solution containing $40 \mathrm{wt} \% \mathrm{C}_{10} \mathrm{E}_{3}$ has been obtained by Oliviero et al. ${ }^{37} \mathrm{We}$ also want to stress that deuterium NMR is a powerful tool for the study of shear-induced changes in lyotropic lamellar systems, as the formation of vesicles has a dramatic effect on the NMR line shape. ${ }^{19,24,43,44}$ Furthermore, it is known from the investigation of phase diagrams of surfactant solutions that the deuterium NMR spectra of water as a solvent are sensitive to a coexistence of structures.

\section{Materials and Methods}

Materials. Triethylene glycol mono- $n$-decyl ether $\left(\mathrm{C}_{10} \mathrm{E}_{3}\right)$ with a purity higher than $99.8 \%$ was purchased from Nikko Chemical Co. (Tokyo, Japan). Deuterium oxide $\left(\mathrm{D}_{2} \mathrm{O}\right)$ was obtained from Sigma

(32) Penfold, J.; Staples, E.; Tucker, I.; Hubbard, J.; Soubiran, L.; Creeth, A Fibre Diffr. Rev. 2003, 11, 68.

(33) Kato, T.; Miyazaki, K.; Kawabata, Y.; Komura, S.; Fujii, S.; Imai, M. J. Phys.: Condens. Matter 2005, 17, S2923.

(34) Fujii, S.; Richtering, W. Eur. Phys. J. E 2006, 19, 139.

(35) Lutti, A.; Callaghan, P. T. J. Magn. Reson. 2007, 187, 251.

(36) Wilkins, G. M. H.; Olmsted, P. D. Eur. Phys. J. E 2006, 21, 133.

(37) Oliviero, C.; Coppola, L.; Gianferri, R.; Nicotera, I.; Olsson, U. Colloids Surf., A 2003, 228, 85 .

(38) Nettesheim, F.; Zipfel, J.; Olsson, U.; Renth, F.; Lindner, P.; Richtering, W. Langmuir 2003, 19, 3603.

(39) Medronho, B.; Fujii, S.; Richtering, W.; Miguel, M. G.; Olsson, U. Colloid Polym. Sci. 2005, 284, 317.

(40) Medronho, B.; Miguel, M. G.; Olsson, U. Langmuir 2007, 23, 5270.

(41) Kleinschmidt, F. Ph.D. Thesis, Albert Ludwig University, Freiburg, Germany, 2005.

(42) Porte, G.; Berret, J.-F.; Harden, J. L. J. Phys. II (France) 1997, 7, 459.

(43) Douliez, J. P.; Bellocq, A. M.; Dufourc, E. J. J. Chim. Phys. Phys.-Chim. Biol. 1994, 91, 874 .

(44) Auguste, F.; Douliez, J. P.; Bellocq, A. M.; Dufourc, E. J. Langmuir 1997, 13, 666.
Chemicals (Steinheim, Germany). Samples containing 40 wt \% surfactant were prepared by weighing the desired amounts of surfactant and water into vials, mixing them in a vortex mixer, and centrifuging them in order to remove air bubbles. All samples were prepared with $\mathrm{D}_{2} \mathrm{O}$ as a probe for deuterium NMR spectroscopy.

Deuterium NMR Spectroscopy. The ${ }^{2} \mathrm{H}$ rheo-NMR experiments were carried out using a cylindrical Couette cell with 14 and $15 \mathrm{~mm}$ inner and outer diameters, respectively. This cell is integrated into an NMR probe for a wide-bore superconducting magnet. The axis of the shear cell is aligned parallel to the external magnetic field. Shear is applied by rotating the outer cylinder with an external motor located below the NMR magnet. The spectra were recorded with a Tecmag Apollo $300 \mathrm{MHz}$ NMR spectrometer operating at the deuterium resonance frequency of $46.073 \mathrm{MHz}$. Spectra were obtained by Fourier transformation of the signal following a single pulse. Typically, 4-16 scans were accumulated for each spectrum, and a recycle delay of $1 \mathrm{~s}$ was used. The temperature of the sample was controlled using an air-flow system. Under shear, the temperature of the sample remained constant within $\pm 0.5^{\circ} \mathrm{C}$. Due to the air flow, some evaporation of water occurs with time; at $34{ }^{\circ} \mathrm{C}$, the loss of solvent was found to be less than $5 \%$ after $10 \mathrm{~h}$.

For each series of experiments, reproducible initial conditions were achieved by filling the sample into the shear cell and increasing the temperature until the isotropic signal of the sponge phase was reached. The sample homogenization and adjustment of the magnetic field homogeneity ("shimming") took place at around $49^{\circ} \mathrm{C}$ under shear at a constant rate of $10 \mathrm{~s}^{-1}$. Under these conditions, the sponge phase, $\mathrm{L}_{3}$, is stable and the typical ${ }^{2} \mathrm{H}$ NMR fingerprint is a narrow singlet. Afterward, the temperature was decreased slowly $\left(0.5^{\circ} \mathrm{C} /\right.$ min) to $42{ }^{\circ} \mathrm{C}$. The temperature must be decreased slowly in order to let the lamellar phase nucleate from the sponge phase. If the temperature is changed too quickly from $49{ }^{\circ} \mathrm{C}\left(\mathrm{L}_{3}\right.$ phase) to $42^{\circ} \mathrm{C}$ ( $\mathrm{L}_{\alpha}$ phase), a phase separation can occur, leading to a coexistence of both phases in the shear cell for an inconveniently long period.

The ${ }^{2} \mathrm{H}$ NMR technique probes the motionally averaged electric quadrupole couplings between the deuterium nuclei ( $\operatorname{spin} I=1$ ) and the electric field gradients at the sites of the observed nuclei. ${ }^{45}$ The residual couplings arise from the anisotropy of the rotational motions of the water molecules and thus depend on the curvature of the hydrophobic/hydrophilic interface ${ }^{24,43,44,46,47}$ When $\mathrm{D}_{2} \mathrm{O}$ molecules experience a macroscopically anisotropic environment, the quadrupolar interaction has a nonzero average, leading to a splitting of the resonance. In the case of uniaxial symmetry, as for the $\mathrm{L}_{\alpha}$ phase, the ${ }^{2} \mathrm{H}$ spectrum consists of a doublet with a frequency separation $\Delta v$ given by ${ }^{45,48}$

$$
\Delta v=\frac{3}{4} \delta\left(\cos ^{2} \theta-1\right)
$$

where $\theta$ is the angle between the director (the symmetry axis of the phase) and the magnetic field and $\delta=e^{2} q Q / h$ is the motionally averaged quadrupole coupling constant. According to eq 1 , the deuterium NMR line shape depends on the distribution of director orientations. A simple doublet is observed only if the lamellar phase has been well aligned, for example, by the action of the magnetic field or by the application of shear. A disordered lamellar phase consisting of many domains of extended flat layers whose orientations are isotropically distributed in space gives rise to the characteristic line shape of a polycrystalline sample also known as powder or Pake pattern.

Translational diffusion in lamellar phases is highly anisotropic and restricted mainly to a given layer. Therefore, diffusion in the MLV state depends on the onion diameter, ${ }^{41}$ and, as demonstrated recently by Lutti and Callaghan, pulsed gradient NMR diffusometry can be employed to determine the dimensions of MLVs in situ under

(45) Abragam, A. Principles of Nuclear Magnetism; Clarendon: Oxford, 1961. (46) Baciu, M.; Olsson, U.; Leaver, M.; Holmes, M. J. Phys. Chem. B 2006, $110,16$.

(47) Gotter, M.; Strey, R.; Olsson, U.; Wennerstrom, H. Faraday Discuss. 2005, 129, 327.

(48) Halle, B.; Wennerstrom, H. J. Chem. Phys. 1981, 75, 1928. 
shear. ${ }^{35}$ Diffusion is also responsible for the dependence of the NMR line shape on the MLV size. As long as the lamellae are planar, the diffusive motion of the probe molecule has no extra rotational component besides the anisotropic local tumbling and the characteristic quadrupole coupling of the $\mathrm{L}_{\alpha}$ phase is not affected. However, when the layers are curved, diffusion along the layers is connected to a rotation and therefore leads to an additional motional narrowing of the spectrum. This results in a decrease and eventually a collapse of the quadrupolar splitting. For our purposes, the spectrum profile gives the information we are looking for because the doublet of the planar lamellae turns into a broad single peak when multilamellar vesicles are formed by shaking or shearing the sample. ${ }^{19,24,35,43}$ For multilamellar vesicles with a typical radius of a few micrometers, a distribution of averaged quadrupole couplings is expected: while diffusion in the inner shells leads to isotropic averaging, diffusion in the outer shells covers only part of the sphere so that a residual coupling results. Thus, the result is a superposition of spectra with different couplings yielding the observed relatively broad but featureless lines. Smaller vesicles lead to narrower bands; an empirical relationship between vesicle radius and line width has been determined for $\mathrm{C}_{10} \mathrm{E}_{3}$ and $\mathrm{C}_{12} \mathrm{E}_{4} \cdot{ }^{41}$ When the two morphologies of the lamellar phase (aligned planar lamellae and MLVs) coexist, the spectrum consists of a superposition of a central singlet originating from the MLVs and a doublet arising from the aligned lamellar phase.

\section{Results and Discussion}

From Planar Lamellae to MLVs: Strain-Controlled Continuous Transformation. The shear diagram of Oliviero et al. ${ }^{37}$ of the aqueous solution containing $40 \mathrm{wt} \% \mathrm{C}_{10} \mathrm{E}_{3}$ was used as a guide for the experiments. In this diagram, the stability regions of MLVs and planar layers are represented as a function of shear rate and temperature. MLVs are stable at temperatures below approximately $40{ }^{\circ} \mathrm{C}$ at sufficiently high shear rates. At higher temperatures and lower shear rates, aligned layers are observed. The two regimes are separated by a transition region, in which the transition from layers to MLVs has been reported to be incomplete. ${ }^{37}$ We performed start-up experiments at a particular temperature and shear rate in one region of the shear diagram, starting from a kinetically stable initial state prepared in the other regime of the shear diagram, and observed the transformation of the lamellar phase, which was monitored by measuring deuterium NMR spectra as a function of time. For all experiments, arrows are shown in the shear diagram merely to indicate the temperatures and shear rates of the initial and final states. In practice, all experiments involving a change of temperature pass through a state of zero shear. Initial and final states within the transition regime of the shear diagram were avoided in order to keep the NMR line shapes as simple as possible.

For experiments involving a temperature decrease, that is, a negative change of temperature, the procedure was the following: After the slow formation of the aligned lamellar phase at $42{ }^{\circ} \mathrm{C}$ (described in the experimental section), a shear rate of $10 \mathrm{~s}^{-1}$ was applied for $30 \mathrm{~min}$. This results in planar layers oriented with their normal in the gradient direction ( $\mathrm{C}$ orientation). The shear was then stopped, and the temperature decreased to a temperature within the stability region of MLVs in the shear diagram. Finally, the shear rate was set at $10 \mathrm{~s}^{-1}$ and ${ }^{2} \mathrm{H}$ NMR spectra were recorded as a function of time. The results of two such experiments, showing the transformation from layers to MLVs at 34 and $25^{\circ} \mathrm{C}$, are depicted in Figures 1 and $2 \mathrm{a}$, respectively. For the lower temperature, additional results from a rheological experiment are shown for comparison in Figure $2 b$. Selected spectra from the two experiments at 25 and $34{ }^{\circ} \mathrm{C}$ can be seen in Figure 3a. In Figure 3b, we have plotted how the quadrupolar splitting (peak-peak separation) evolves as a function of strain.

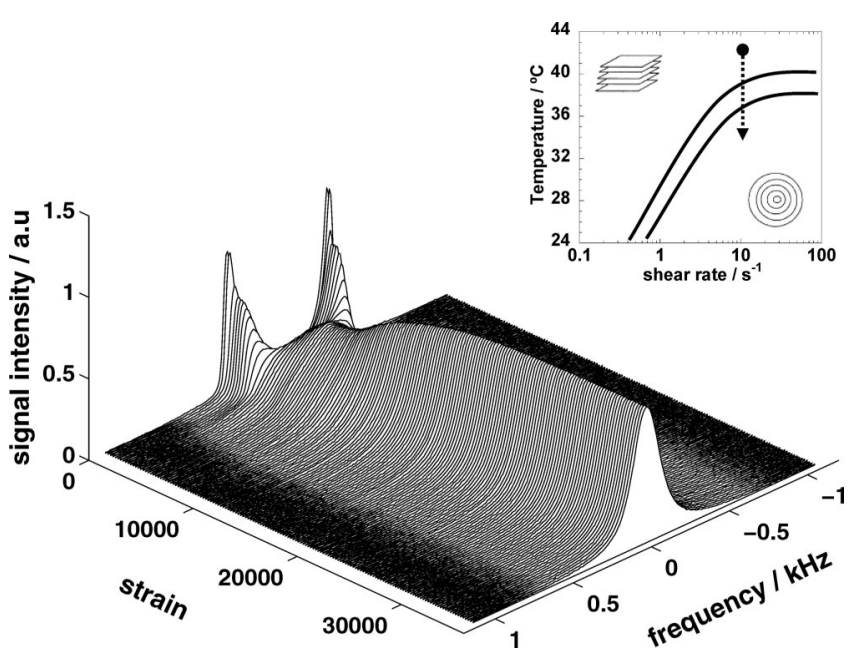

Figure 1. Strain evolution of spectra during the transformation from planar layers to MLVs at $34{ }^{\circ} \mathrm{C}$ and a constant shear rate of $10 \mathrm{~s}^{-1}$. Spectra were recorded every $24 \mathrm{~s}$ up to $3600 \mathrm{~s}$ (36 000 strain units). The inset shows the shear diagram of the $\mathrm{C}_{10} \mathrm{E}_{3} / \mathrm{D}_{2} \mathrm{O}$ system and the stability regions of MLVs and planar layers as a function of temperature and shear rate. The arrow represents the conditions at which the initial state was prepared and the conditions of the start-up experiment.

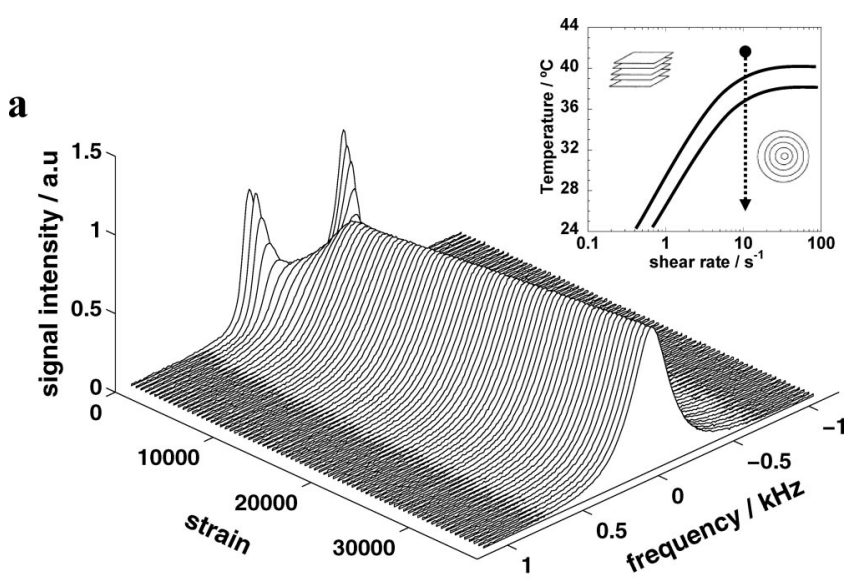

b

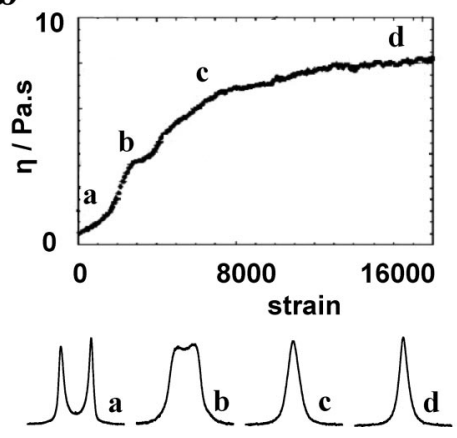

Figure 2. (a) Strain evolution of spectra during the transformation from planar layers to MLVs at $25{ }^{\circ} \mathrm{C}$ and a constant shear rate of $10 \mathrm{~s}^{-1}$. Spectra were recorded every $12 \mathrm{~s}$ (only every second spectrum is shown) up to $t=3600 \mathrm{~s}$ (36000 strain units). (b) Viscosity versus time curve during the MLV formation at $10 \mathrm{~s}^{-1}$. The ${ }^{2} \mathrm{H}$ NMR fingerprints of the initial, intermediate, and final structures are also shown.

At both temperatures, a gradual change of the line shapes with increasing strain, $\gamma=\dot{\gamma} t$, can be seen, suggesting that the transformation from layers to vesicles is a continuous process, in agreement with previous observations..$^{8,17,19,38}$ The line shape evolves in two ways; the resonances are broadened and the quadrupolar splitting decreases. The decrease in quadrupolar 

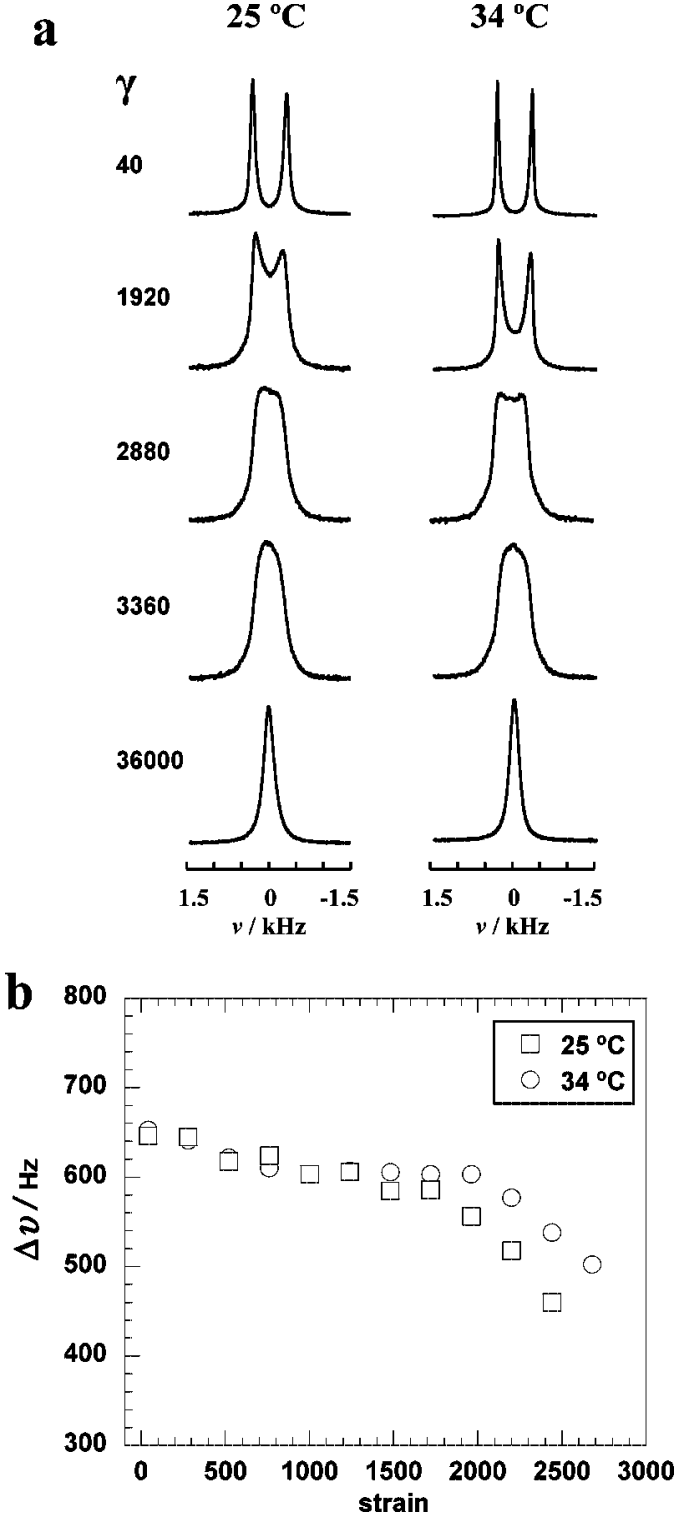

Figure 3. (a) Selected NMR spectra from Figures 1 and 2a. (b) Initial evolution of the quadrupolar splitting as a function of applied strain at 25 and $34{ }^{\circ} \mathrm{C}$.

splitting indicates that we introduce more curved structures allowing for an additional averaging of the quadrupolar interaction. The line broadening indicates that the molecular reorientation motion, resulting in the reduced splitting, is slow. It is likely that this reorientational motion involves lateral diffusion of water molecules within curved water-film domains, where the curvature still is low, making the reorientational motion slow. Zipfel et al. ${ }^{29}$ have suggested, based on small angle neutron and light scattering data, that the planar bilayers initially transformed into some cylindrical structure such as, for example, multilamellar cylinders. The formation of this intermediate structure is completed after 3000 strain units. ${ }^{29}$ For such a cylindrical structure, we expect a quadrupolar splitting which is half of the splitting in the planar lamellar phase, unless the cylinder radius is too large. For large diameter (micrometer) cylinders, we do expect a broadening of the resonances. However, if the cylinder diameter is too large, there will not be complete angular averaging within the cylindrical water layer which has to occur on a time scale that is shorter than the inverse of the lamellar phase quadrupolar splitting. As can be seen in Figure 3b, the data show a reduction of the quadrupolar splitting that approaches ap- proximately half of the initial value as the strain approaches 3000 strain units. Due to the line broadening, we could not follow the evolution of the quadrupolar splitting further than what is shown in Figure 3b. We conclude, however, that the data are consistent with the suggestion of Zipfel et al. ${ }^{29}$ that the planar bilayers, oriented with the normal in the gradient direction (C orientation), initially are bent and transformed into cylindrical structures.

A "two-dimensional powder" consisting of large domains of planar layers, the normal vectors of which are distributed in the plane perpendicular to the flow, a structure also consistent with the previously reported scattering pattern of the intermediate state, ${ }^{29}$ would yield a spectrum consisting of a weighted sum of doublets with splittings according to eq 1 , for values of $\theta$ ranging from $0^{\circ}$ to $90^{\circ}$. The NMR spectrum of such a two-dimensional powder has maxima at the frequencies of the initial peaks (layers aligned with their normal at $\theta=90^{\circ}$ with respect to the magnetic field) and at twice these frequencies $\left(\theta=0^{\circ}\right)$. This is clearly not observed; hence, this type of structure can be ruled out.

In Figure $2 b$, the characteristic NMR spectra obtained at different stages of the layer-to-MLV transformation are correlated with the viscosity data (from ref 28). The major changes of the line shapes occur in the same range of strain where the strongest increase of viscosity is observed, while there is little change in the line shape as the final plateau of the viscosity is reached. Spectrum b corresponds approximately to 3000 strain units at which the viscosity curve displays a narrow plateau.

As can be seen from the individual spectra shown in Figure 3a, the single peak characteristic of the final MLV state does not coexist with the initial doublet. Eventually, the lamellar doublet is no longer resolved and a broad single peak is observed. The single peak undergoes a continuous change of shape, and its width decreases with strain, indicating that the size of the structures is getting smaller, until a final steady state is reached. A separation of the spectra into different components, one corresponding to aligned layers plus another one corresponding to vesicles, is not possible. This indicates that the sample remains homogeneous in space, as opposed to a two-state coexistence, throughout the transformation process. The continuous change of the line shape and the initial decrease of the splitting suggest that defects are being formed in the entire sample until the structure of monodisperse MLVs is fully developed.

Earlier results for the $\mathrm{C}_{10} \mathrm{E}_{3}$ /water system have shown that the shear-induced formation of MLVs is a strain-controlled process. $^{29,38}$ The comparison of the layer-to-MLV transformation of $\mathrm{C}_{10} \mathrm{E}_{3}$ at $25{ }^{\circ} \mathrm{C}$ for different shear rates has shown that corresponding structures occur at the same strain. Here, we focus on the temperature dependence of strain scaling, which was briefly mentioned to be very similar at 25 and $32{ }^{\circ} \mathrm{C}$, whereas a different transition behavior was observed at $38^{\circ} \mathrm{C} .{ }^{38}$ In Figure 3, spectra obtained at the same strain $\gamma$ during the two experiments at 25 and $34^{\circ} \mathrm{C}$ are shown. The comparison of these spectra illustrates the similarity of the line shapes measured at the same strain. This demonstrates that the transition process occurs approximately on the same strain scale, even for the different temperatures of 25 and $34{ }^{\circ} \mathrm{C}$. Thus, NMR shows that, within this range, temperature apparently has no large influence on the kinetics of the MLV formation, in agreement with the previous scattering results. $^{38}$

It is important to mention that we found the same type of continuous mechanism when starting from a lamellar phase previously oriented by applying a low shear rate at lower temperature, within the region of the aligned $L_{\alpha}$ phase in the shear diagram, and then increasing the shear rate suddenly at 

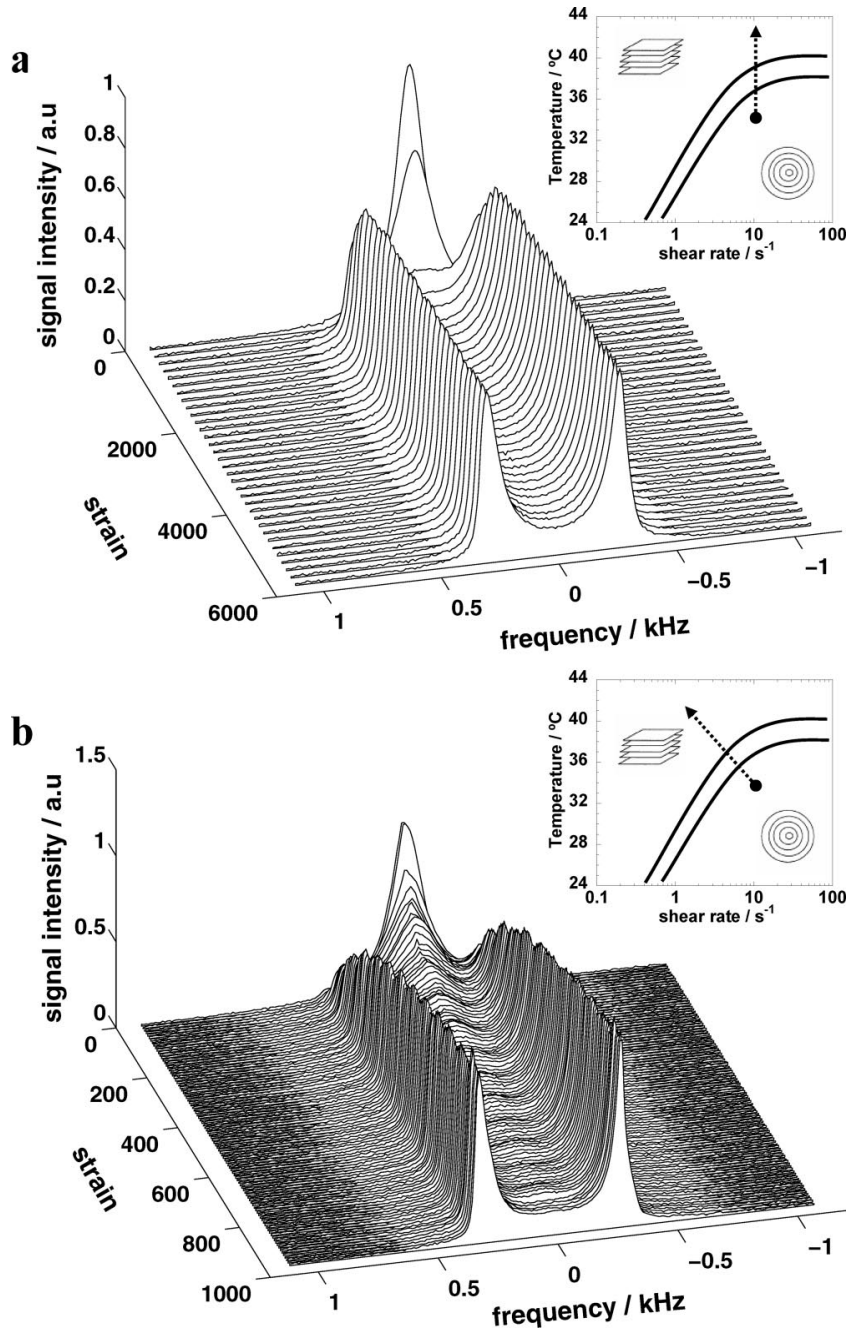

Figure 4. (a) Strain evolution of spectra during the transformation from MLVs to planar lamellae at $42^{\circ} \mathrm{C}$ and $\dot{\gamma}=10 \mathrm{~s}^{-1}$. Spectra were recorded every $20 \mathrm{~s}$ up to $t=600 \mathrm{~s}$ (6000 strain units). (b) Strain evolution of spectra during the transformation from MLVs to planar lamellae at 42 ${ }^{\circ} \mathrm{C}$ and $\dot{\gamma}=1 \mathrm{~s}^{-1}$. Spectra were recorded every $10 \mathrm{~s}$ up to $t=1000 \mathrm{~s}$ (1000 strain units). The insets show the locations of the initial and final states.

constant temperature. Again, a continuous transformation is observed by following the NMR spectra as a function of time. Our observations of the transformation from layers to MLVs thus agree with the previously reported continuous nature of this transition. ${ }^{8,17,19}$ For the $\mathrm{C}_{10} \mathrm{E}_{3} /$ water system, in particular, the continuous evolution of the morphology is also evident in the small angle neutron and light scattering patterns. ${ }^{38}$

From MLVs to Planar Lamellae: Strain-Controlled Nucleation and Growth. After the formation of MLVs at $34{ }^{\circ} \mathrm{C}$, shown in Figure 1, the shear was stopped and the temperature increased to $42{ }^{\circ} \mathrm{C}$, where MLVs are not stable under shear. After a few minutes of thermal equilibration (where the MLV structure remained without significant changes in the ${ }^{2} \mathrm{H}$ NMR spectrum), shear was started again at a rate of $10 \mathrm{~s}^{-1}$ and spectra were recorded every $20 \mathrm{~s}$. The collection of the ${ }^{2} \mathrm{H}$ NMR spectra of this start-up experiment, in which we now start from an initial state prepared within the stability regime of vesicles under shear and observe their destruction at increased temperature, is presented in Figure 4a. These data show that the most important part of the transformation occurs in a couple of seconds, and the few spectra measured during the transformation do not characterize the evolution of this transition very well. The kinetics of the

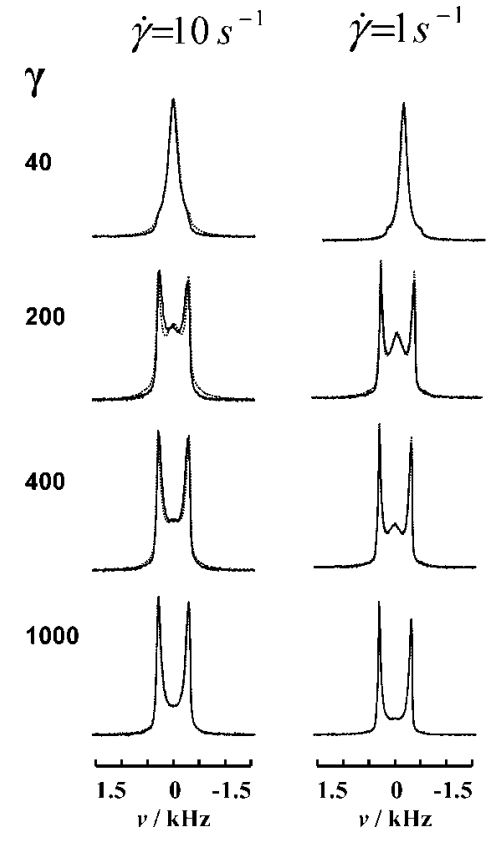

Figure 5. Selected NMR spectra from Figure 4 (solid lines) and their corresponding best fits (dashed lines) obtained by the weighted superposition of the spectra of the two components.

destruction of MLVs and the formation of planar lamellae, under these conditions, is too fast to be followed in detail.

In order to slow down the transition, an additional start-up experiment was carried out at a lower shear rate of $\dot{\gamma}=1 \mathrm{~s}^{-1}$ while keeping the same temperature $\left(42^{\circ} \mathrm{C}\right)$. The preparation of the initial state was the same (first, the sample orientation at 42 ${ }^{\circ} \mathrm{C}$ and $\dot{\gamma}=10 \mathrm{~s} \mathrm{~s}^{-1}$, followed by MLV formation at $34{ }^{\circ} \mathrm{C}$ and $\dot{\gamma}=10 \mathrm{~s}^{-1}$ ). As shown in Figure $4 \mathrm{~b}$, the transition from MLVs to planar lamellae can now be observed in detail and it is possible to follow the initial structural transformations. The spectra were recorded every $10 \mathrm{~s}$. The line shapes suggest a discontinuous transition, with a coexistence regime of both structures. In contrast to the transition in the opposite direction discussed above, in Figure 4 the spectrum of the final state of aligned layers occurs almost immediately at very low strain. The quadrupolar splitting changes very little during the transition (less than 5\%). It must be noted that the full splitting of the aligned $\mathrm{L}_{\alpha}$ phase is observed only when the domains of planar layers are large enough for the water molecules to stay within one domain during the time scale of the NMR measurement. If the lateral dimension of a stack of planar layers is smaller than a few micrometers, the water molecules can diffuse through defects into neighboring MLV domains and the quadrupolar splitting is reduced. Thus, the observed slight increase of the quadrupolar splitting is consistent with the growth of the domains consisting of planar layers.

Selected spectra of both the experiments shown in Figure 4 are presented in Figure 5. Since the transition is discontinuous, passing through a state of two coexisting structures, it is possible to separate the spectra into different components. In general, the area under an NMR spectrum is proportional to the concentration of molecules in a particular state. By fitting the spectra with a weighted sum of the spectra of each component, we can determine the amount of each component. The spectrum of the MLV component can be described as a Lorentzian function: ${ }^{45}$

$$
I(v)=\frac{I^{\max }}{1+\left(2\left(v-v_{0}\right) / \Delta v_{1 / 2}\right)^{2}}
$$

Here, $v$ is the frequency, $v_{0}$ is the peak center, $I^{\max }$ is the peak maximum, and $\Delta v_{1 / 2}$ is the peak width at half-height. The line 

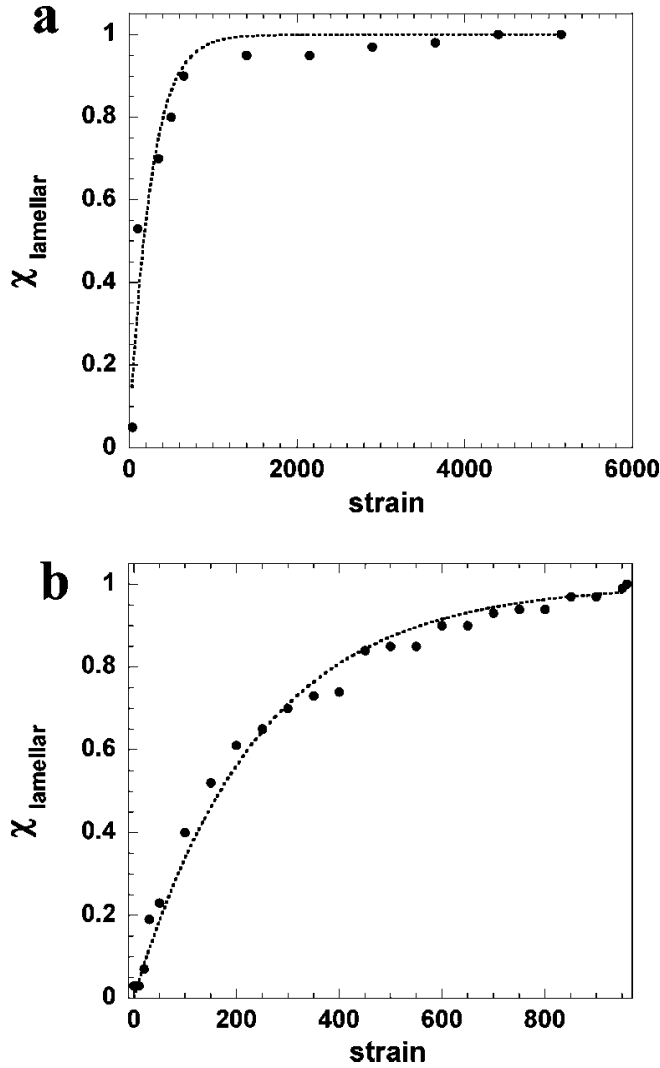

Figure 6. Strain evolution of the planar lamellar fraction, $\chi_{\text {lamellar, }}$ at 42 ${ }^{\circ} \mathrm{C}$ obtained from fitting the spectra as shown in Figure 5. The dotted lines are fitted curves according to eq 3 with (a) $k^{-1}=252$ strain units for shear rate $10 \mathrm{~s}^{-1}$ and (b) $k^{-1}=242$ strain units for shear rate $1 \mathrm{~s}^{-1}$.

shape of the doublet arising from the planar layers, on the other hand, shows a rather large intensity in the center of the spectrum and is not well represented by a sum of two Lorentzians. Therefore, instead of using a sum of three Lorentzians as a theoretical model function to fit the experimental spectra, we tried to fit a superposition of the first and the last experimental spectra to the intermediate ones. However, this procedure did not yield satisfactory results, since the width of the MLV peak changes initially due an increase of the vesicle size. Therefore, a combination of the two approaches was chosen for the fitting of the first few spectra, measured at low strains: the MLV component was represented by a Lorentzian model function of variable width, while the component of planar layers was represented by its experimental line shape measured at large strain. The fitted Lorentzian shape of the last spectrum treated in this way was then used as the MLV component in the fitting of all later spectra. In Figure 5, the experimental line shapes are shown together with the corresponding best fits. The decay of the central peak (MLVs) as well as the rise of the doublet intensity (planar layers) can be recognized.

The fraction of the planar layers obtained by fitting the line shapes is shown in Figure 6 as a function of strain. The observed discontinuity of the transformation from MLVs to planar layers suggests a nucleation and growth process. By applying an exponential function of the form

$$
\chi_{\text {lamellar }}=1-\exp (-k \gamma)
$$

reasonable fits describing the growth of the fraction of the lamellar component, $\chi_{\text {lamellar, }}$ with increasing strain can be obtained. Strain $\gamma=\dot{\gamma} t$ instead of time $t$ is used as the variable in eq 3 in order
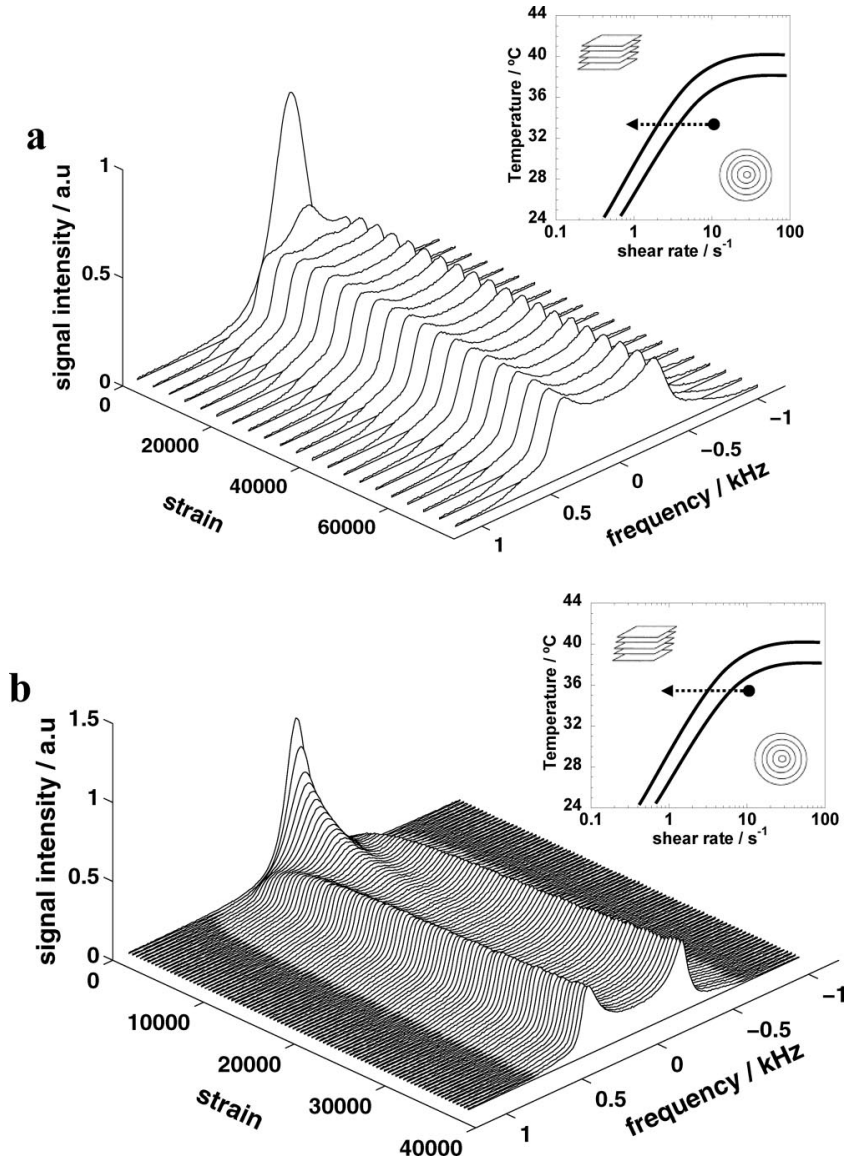

Figure 7. (a) Strain evolution of the spectra during the transformation from MLVs to planar lamellae at $34{ }^{\circ} \mathrm{C}$ and $\dot{\gamma}=1 \mathrm{~s}^{-1}$. Spectra were recorded every hour up to $t=20 \mathrm{~h}$ (72000 strain units). (b) Strain evolution of the spectra during the transformation from MLVs to planar layers at $36^{\circ} \mathrm{C}$ and $\dot{\gamma}=1 \mathrm{~s}^{-1}$. Spectra were recorded every 5 min up to $t=660 \mathrm{~min}$ (39 600 strain units). The insets show the locations of the initial and final states.

to analyze whether strain-scaling, as found for the formation of MLVs from aligned layers, applies to the reverse transition as well. From the phenomenological fits, shown as dotted lines in Figure 6, values for the characteristic strain $k^{-1}$ of 252 and 242 strain units are determined for the MLV-to-layer transformations at 10 and $1 \mathrm{~s}^{-1}$, respectively. The characteristic strains are essentially the same, showing that the transformation process scales with the applied strain.

The transition from MLVs to planar lamellae was also probed by a start-up experiment in which only the shear rate was changed from $\dot{\gamma}=10 \mathrm{~s}^{-1}$ for the initial state to $\dot{\gamma}=1 \mathrm{~s}^{-1}$ for the final state but the temperature was kept constant at $34{ }^{\circ} \mathrm{C}$. In Figure $7 \mathrm{a}$, the corresponding collection of spectra, one recorded every hour, is depicted. Under these conditions, the transformation process is very slow and even for a strain larger than 72000 (after more than $20 \mathrm{~h}$ ) no steady state seems to have been reached yet. The pattern observed is not a simple doublet characteristic of an aligned sample but the Pake pattern of a disordered sample showing the characteristic "shoulders" corresponding to $\theta=0^{\circ}$. There are two possible explanations for the observed line shape. It may result from the superposition of doublets due to different lamellar orientations that are formed during the destruction of MLVs. As shown in the inset in Figure 7a, the shear rate change ended in a position near the border of the transition region, determined by Oliviero et al., ${ }^{37}$ where the structure has not been clearly identified. Therefore, the Pake spectra could, in principle, be related to huge MLVs. In order to obtain a rotational diffusion 
constant $D_{\text {rot }}$ of $\mathrm{D}_{2} \mathrm{O}$ large enough to observe a Pake pattern, a vesicle diameter of about $100 \mu \mathrm{m}$ or more would be necessary.

With the final state of the previously described experiment being very close to the transition region, no doublet of aligned layers could be observed. Therefore, another experiment was carried out in which MLVs were prepared at a higher temperature, $36^{\circ} \mathrm{C}$, and the same change in $\dot{\gamma}$, from 10 to $1 \mathrm{~s}^{-1}$, was applied. At this temperature, the final position in the shear diagram is far enough away from the transition region. In Figure 7b, the strain evolution of the spectra (recorded every $5 \mathrm{~min}$ ) can be observed. At $36{ }^{\circ} \mathrm{C}$, a discontinuous transition (with a coexistence of both structures) is again observed, and at a strain of 36000 (after $10 \mathrm{~h}$ ) the oriented lamellar phase was recovered. However, low shoulders can be seen in the final spectrum, indicating that under the applied conditions of temperature and shear rate the steady state consists of planar layers which are less well aligned compared to those at $42{ }^{\circ} \mathrm{C}$. A similar incomplete alignment of layers has been observed for $\mathrm{C}_{12} \mathrm{E}_{4}$ in cone-and-plate geometry at low shear rates below the threshold where MLVs are formed. ${ }^{19}$ For the spectra shown in Figure $7 \mathrm{~b}$, the quadrupolar splitting $\Delta v$ changed by about $5 \%$, similar to what was observed for the MLV-to-layer transition at $42{ }^{\circ} \mathrm{C}$ and $\gamma=1 \mathrm{~s}^{-1}$ (cf. Figure 4b). The increase of the splitting is again consistent with a growth of the layer domains, but there may be an additional contribution due to some water evaporation, since the experiment ran for more than $11 \mathrm{~h}$. Selected spectra from Figure 7 at the same strain are shown in Figure 8. Despite the fact that the final state of the two experiments at 34 and $36{ }^{\circ} \mathrm{C}$ is obviously different, the trends of the spectra during the formation of MLVs are similar. From the analysis of the kinetics of the transformation at $36^{\circ} \mathrm{C}$, a characteristic strain $k^{-1}$ of 1890 strain units was obtained, showing that the process is much slower than the vesicle destruction observed at $42{ }^{\circ} \mathrm{C}$.

\section{Conclusions}

The transition from planar layers to MLVs and in the reverse direction was followed in the $\mathrm{C}_{10} \mathrm{E}_{3} / \mathrm{D}_{2} \mathrm{O}$ system by rheo-NMR spectroscopy. Most importantly, two different mechanisms are found, corresponding to either a continuous or a discontinuous transformation. The nature of the transitions does not depend on whether the shear rate or the temperature is changed but only on the direction of the transition. ${ }^{49}$

The transformation from planar layers to onions was found to be continuous and strain-controlled, in agreement with previous results. The transition in this direction not only shows strainscaling for different shear rates at constant temperature ${ }^{29,38}$ but

(49) One of the reviewers suggested a possible explanation for the difference of the two transition directions: When one moves towards the thermodynamically stable structure, that is, the equilibrium structure at zero shear, a discontinuous behavior, akin to a first-order phase transition, is found, whereas the observed continuous MLV formation is symptomatic of the kinetic processes associated with a mechanically driven structure formation.

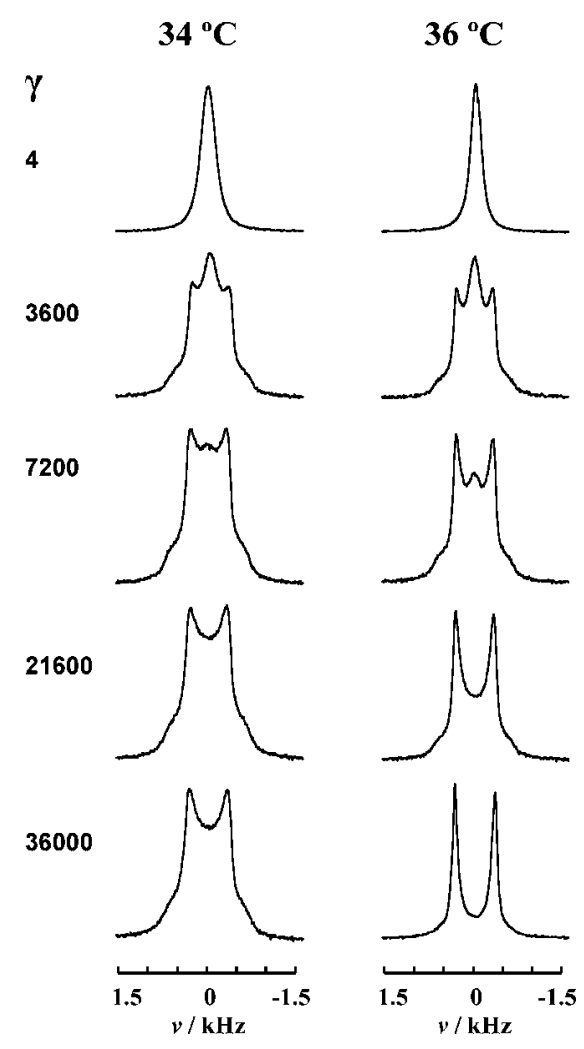

Figure 8. Selected NMR spectra from Figure 7.

also exhibits approximately the same dependence on strain for different temperatures $\left(25\right.$ and $\left.34{ }^{\circ} \mathrm{C}\right)$.

In contrast, the reverse transition from MLVs to layers, which had not been investigated in detail before, is a discontinuous process of nucleation and growth. Strain scaling is found for the reverse transition as well, at least for shear rates of 1 and $10 \mathrm{~s}^{-1}$ at a constant temperature of $42^{\circ} \mathrm{C}$. However, when the temperature is lowered to $36{ }^{\circ} \mathrm{C}$, the reverse transition occurs with a characteristic strain which is one order of magnitude larger; in other words, the transition becomes slower when the temperature is decreased but the strain rate is kept constant.

Acknowledgment. This work was supported by grants from the Swedish Research Council, the Swedish Foundation for International Cooperation in Research and Higher Education (STINT), and the German Academic Exchange Service (DAAD). The Colloid Group in Coimbra University is supported by grants from the Fundação para a Ciência e Tecnologia (FCT) (Projects Ref. POCTI/QUI/45344/2002 and POCTI/QUI/58689/2004). B.M. thanks Fundação para a Ciência e tecnologia (FCT) (Project Ref. SFRH/BD/21467/2005).

LA800326A 